\title{
A new contraction mapping principle in partially ordered metric spaces and applications to ordinary differential equations
}

\author{
Fangfang Yan, Yongfu Su* and Qiansheng Feng
}

${ }^{*}$ Correspondence:

suyongfu@gmail.com Department of Mathematics, Tianjin

Polytechnic University, Tianjin, 300387, P.R. China

\begin{abstract}
The aim of this paper is to extend the results of Harjani and Sadarangani and some other authors and to prove a new fixed point theorem of a contraction mapping in a complete metric space endowed with a partial order by using altering distance functions. Our theorem can be used to investigate a large class of nonlinear problems. As an application, we discuss the existence of a solution for a periodic boundary value problem.
\end{abstract}

Keywords: contraction mapping principle; partially ordered metric spaces; fixed point; altering distance function; differential equation

\section{Introduction}

The Banach contraction principle is a classical and powerful tool in nonlinear analysis. Weak contractions are generalizations of Banach's contraction mapping studied by several authors. In [1-8], the authors prove some types of weak contractions in complete metric spaces respectively. In particular, the existence of a fixed point for weak contraction and generalized contractions was extended to partially ordered metric spaces in [2, 9-18]. Among them, the altering distance function is basic concept. Such functions were introduced by Khan et al. in [1], where they present some fixed point theorems with the help of such functions. Firstly, we recall the definition of an altering distance function.

Definition 1.1 An altering distance function is a function $\psi:[0, \infty) \rightarrow[0, \infty)$ which satisfies

(a) $\psi$ is continuous and nondecreasing.

(b) $\psi=0$ if and only if $t=0$.

Recently, Harjani and Sadarangani proved some fixed point theorems for weak contraction and generalized contractions in partially ordered metric spaces by using the altering distance function in $[11,19]$ respectively. Their results improve the theorems of $[2,3]$.

Theorem 1.1 [11] Let $(X, \leq)$ be a partially ordered set, and suppose that there exists a metric $d \in X$ such that $(X, d)$ is a complete metric space. Let $f: X \rightarrow X$ be a continuous and nondecreasing mapping such that

$$
d(f(x), f(y)) \leq d(x, y)-\psi(d(x, y)) \quad \text { for } x \geq y,
$$

(c) 2012 Yan et al.; licensee Springer. This is an Open Access article distributed under the terms of the Creative Commons Attribution License (http://creativecommons.org/licenses/by/2.0), which permits unrestricted use, distribution, and reproduction in any medium, provided the original work is properly cited. 
where $\psi:[0, \infty) \longrightarrow[0, \infty)$ is continuous and nondecreasing function such that $\psi$ is positive in $(0, \infty), \psi(0)=0$ and $\lim _{t \rightarrow \infty} \psi(t)=\infty$. If there exists $x_{0} \in X$ with $x_{0} \leq f\left(x_{0}\right)$, then $f$ has a fixed point.

Theorem 1.2 [19] Let $(X, \leq)$ be a partially ordered set, and suppose that there exists a metric $d \in X$ such that $(X, d)$ is a complete metric space. Let $f: X \rightarrow X$ be a continuous and nondecreasing mapping such that

$$
\psi d(f(x), f(y)) \leq \psi(d(x, y))-\phi(d(x, y)) \quad \text { for } x \geq y
$$

where $\psi$ and $\phi$ are altering distance functions. If there exists $x_{0} \in X$ with $x_{0} \leq f\left(x_{0}\right)$, then $f$ has a fixed point.

Subsequently, Amini-Harandi and Emami proved another fixed point theorem for contraction type maps in partially ordered metric spaces in [10]. The following class of functions is used in [10].

Let $\Re$ denote the class of functions $\beta:[0, \infty) \longrightarrow[0,1)$ which satisfies the condition $\beta\left(t_{n}\right) \longrightarrow 1 \Rightarrow t_{n} \longrightarrow 0$.

Theorem 1.3 [10] Let $(X, \leq)$ be a partially ordered set, and suppose that there exists a metric $d$ such that $(X, d)$ is a complete metric space. Let $f: X \rightarrow X$ be an increasing mapping such that there exists an element $x_{0} \in X$ with $x_{0} \leq f\left(x_{0}\right)$. Suppose that there exists $\beta \in \Re$ such that

$$
d(f(x), f(y)) \leq \beta(d(x, y)) d(x, y) \quad \text { for each } x, y \in X \text { with } x \geq y .
$$

Assume that either $f$ is continuous or $M$ is such that if an increasing sequence $x_{n} \rightarrow x \in X$, then $x_{n} \leq x, \forall n$. Besides, iffor each $x, y \in X$, there exists $z \in m$ which is comparable to $x$ and $y$, then $f$ has a unique fixed point.

The purpose of this paper is to extend the results of $[10,11,19]$ and to obtain a new contraction mapping principle in partially ordered metric spaces. The result is more generalized than the results of $[10,11,19]$ and other works. The main theorems can be used to investigate a large class of nonlinear problems. In this paper, we also present some applications to first- and second-order ordinary differential equations.

\section{Main results}

We first recall the following notion of a monotone nondecreasing function in a partially ordered set.

Definition 2.1 If $(X, \leq)$ is a partially ordered set and $T: X \rightarrow X$, we say that $T$ is monotone nondecreasing if $x, y \in X, x \leq y \Rightarrow T(x) \leq T(y)$.

This definition coincides with the notion of a nondecreasing function in the case where $X=R$ and $\leq$ represents the usual total order in $\mathrm{R}$.

We shall need the following lemma in our proving. 
Lemma 2.1 If $\psi$ is an altering distance function and $\phi:[0, \infty) \rightarrow[0, \infty)$ is a continuous function with the condition $\psi(t)>\phi(t)$ for all $t>0$, then $\phi(0)=0$.

Proof Since $\phi(t)<\psi(t)$ and $\phi, \psi$ are continuous, we have

$$
0 \leq \phi(0)=\lim _{t \rightarrow 0} \phi(t) \leq \lim _{t \rightarrow 0} \psi(t)=\psi(0)=0 .
$$

This finishes the proof.

In what follows, we prove the following theorem which is the generalized type of Theorem 1.1-1.3.

Theorem 2.1 Let $X$ be a partially ordered set and suppose that there exists a metric $d$ in $x$ such that $(X, d)$ is a complete metric space. Let $T: X \rightarrow X$ be a continuous and nondecreasing mapping such that

$$
\psi(d(T x, T y)) \leq \phi(d(x, y)), \quad \forall x \geq y,
$$

where $\psi$ is an altering distance function and $\phi:[0, \infty) \rightarrow[0, \infty)$ is a continuous function with the condition $\psi(t)>\phi(t)$ for all $t>0$. If there exists $x_{0} \in X$ such that $x_{0} \leq T x_{0}$, then $T$ has a fixed point.

Proof Since $T$ is a nondecreasing function, we obtain, by induction, that

$$
x_{o} \leq T x_{0} \leq T^{2} x_{0} \leq T^{3} x_{0} \leq \cdots \leq T^{n} x_{0} \leq T^{n+1} x_{0} \leq \cdots
$$

Put $x_{n+1}=T x_{n}$. Then for each integer $n \geq 1$, as the elements $x_{n+1}$ and $x_{n}$ are comparable, from (1) we get

$$
\psi\left(d\left(x_{n+1}, x_{n}\right)\right)=\psi\left(d\left(T x_{n}, T x_{n-1}\right)\right) \leq \phi\left(d\left(x_{n}, x_{n-1}\right)\right) .
$$

Using the condition of Theorem 2.1, we have

$$
d\left(x_{n+1}, x_{n}\right)<d\left(x_{n}, x_{n-1}\right) .
$$

Hence the sequence $d\left(x_{n+1}, x_{n}\right)$ is decreasing, and consequently, there exists $r \geq 0$ such that

$$
d\left(x_{n+1}, x_{n}\right) \rightarrow r,
$$

as $n \rightarrow \infty$. Letting $n \rightarrow \infty$ in (2), we get

$$
\psi(r) \leq \phi(r) .
$$

By using the condition of Theorem 2.1, we have $r=0$, and hence

$$
d\left(x_{n+1}, x_{n}\right) \rightarrow 0,
$$


as $n \rightarrow \infty$. In what follows, we will show that $\left\{x_{n}\right\}$ is a Cauchy sequence. Suppose that $\left\{x_{n}\right\}$ is not a Cauchy sequence. Then, there exists $\varepsilon>0$ for which we can find subsequences $\left\{x_{n_{k}}\right\}$ with $n_{k}>m_{k}>k$ such that

$$
d\left(x_{n_{k}}, x_{m_{k}}\right) \geq \varepsilon
$$

for all $k \geq 1$. Further, corresponding to $m_{k}$, we can choose $n_{k}$ in such a way that it is the smallest integer with $n_{k}>m_{k}$ satisfying (5). Then

$$
d\left(x_{n_{k-1}}, x_{m_{k-1}}\right)<\varepsilon
$$

From (5) and (6), we have

$$
\varepsilon \leq d\left(x_{n_{k}}, x_{m_{k}}\right) \leq d\left(x_{n_{k}}, x_{n_{k-1}}\right)+d\left(x_{n_{k-1}}, x_{m_{k}}\right)<d\left(x_{n_{k}}, x_{n_{k-1}}\right)+\varepsilon
$$

Letting $k \rightarrow \infty$ and using (4), we get

$$
\lim _{k \rightarrow \infty} d\left(x_{n_{k}}, x_{m_{k}}\right)=\varepsilon
$$

By using the triangular inequality, we have

$$
\begin{aligned}
& d\left(x_{n_{k}}, x_{m_{k}}\right) \leq d\left(x_{n_{k}}, x_{n_{k-1}}\right)+d\left(x_{n_{k-1}}, x_{m_{k-1}}\right)+d\left(x_{m_{k-1}}, x_{m_{k}}\right), \\
& d\left(x_{n_{k-1}}, x_{m_{k-1}}\right) \leq d\left(x_{n_{k-1}}, x_{n_{k}}\right)+d\left(x_{n_{k}}, x_{m_{k}}\right)+d\left(x_{m_{k}}, x_{m_{k-1}}\right) .
\end{aligned}
$$

Letting $k \rightarrow \infty$ in the above two inequalities and (4) and (7), we have

$$
\lim _{k \rightarrow \infty} d\left(x_{n_{k-1}}, x_{m_{k-1}}\right)=\varepsilon
$$

As $n_{k}>m_{k}$ and $x_{n_{k-1}}$ and $x_{m_{k-1}}$ are comparable, using (1), we have

$$
\psi\left(d\left(x_{n_{k}}, x_{m_{k}}\right)\right) \leq \phi\left(d\left(x_{n_{k-1}}, x_{m_{k-1}}\right)\right)
$$

Letting $k \rightarrow \infty$ and taking into account (7) and (8), we have

$$
\psi(\varepsilon) \leq \phi(\varepsilon)
$$

From the condition of Theorem 2.1, we get $\varepsilon=0$, which is a contradiction. This shows that $\left\{x_{n}\right\}$ is a Cauchy sequence and, since $X$ is a complete metric space, there exists $z \in X$ such that $x_{n} \rightarrow z$ as $n \rightarrow \infty$. Moreover, the continuity of $T$ implies that

$$
z=\lim _{n \rightarrow \infty} x_{n+1}=\lim _{n \rightarrow \infty} T x_{n}=T z
$$

and this proves that $z$ is a fixed point. This completes the proof. 
In what follows, we prove that Theorem 2.1 is still valid for $T$ not necessarily being continuous, assuming the following hypothesis in $X$ :

$$
\begin{aligned}
& \text { If }\left(x_{n}\right) \text { is a nondecreasing sequence in } X \text { such } \\
& \text { that } x_{n} \rightarrow x \text {, then } x_{n} \leq x \text { for all } n \in N \text {. }
\end{aligned}
$$

Theorem 2.2 Let $(X, \leq)$ be a partially ordered set and suppose that there exists a metric $d$ in $X$ such that $(X, d)$ is a complete metric space. Assume that $X$ satisfies (9). Let $T: X \rightarrow X$ be a nondecreasing mapping such that

$$
\psi(d(T x, T y)) \leq \phi(d(x, y)), \quad \forall x \geq y,
$$

where $\psi$ is an altering distance function and $\phi:[0, \infty) \rightarrow[0, \infty)$ is a continuous function with the conditions $\psi(t)>\phi(t)$ for all $t>0$. If there exists $x_{0} \in X$ such that $x_{0} \leq T x_{0}$, then $T$ has a fixed point.

Proof Following the proof of Theorem 2.1, we only have to check that $T(z)=z$. As $\left(x_{n}\right)$ is a nondecreasing sequence in $X$ and $\lim _{n \rightarrow \infty} x_{n}=z$, the condition (9) gives us that $x_{n} \leq z$ for every $n \in N$, and consequently,

$$
\psi\left(d\left(x_{n+1}, T(z)\right)\right)=\psi\left(d\left(T\left(x_{n}\right), T(z)\right)\right) \leq \phi\left(d\left(x_{n}, z\right)\right) .
$$

Letting $n \rightarrow \infty$ and taking into account that $\psi$ is an altering distance function, we have

$$
\psi(d(z, T(z))) \leq \phi(0)
$$

Using Lemma 2.1, we have $\phi(0)=0$, which implies $\Psi(d(z, T(z)))=0$. Thus $d(z, T(z))=0$ or equivalently, $T(z)=z$.

Now, we present an example where it can be appreciated that the hypotheses in Theorems 2.1 and Theorems 2.2 do not guarantee the uniqueness of the fixed point. The example appears in [17].

Let $X=\{(1,0),(0,1)\} \subset R^{2}$ and consider the usual order $(x, y) \leq(z, t) \Leftrightarrow x \leq z, y \leq t$. Thus, $(x, y)$ is a partially ordered set whose different elements are not comparable. Besides, $\left(X, d_{2}\right)$ is a complete metric space and $d_{2}$ is the Euclidean distance. The identity map $T(x, y)=(x, y)$ is trivially continuous and nondecreasing, and the condition (9) of Theorem 2.2 is satisfied since the elements in $X$ are only comparable to themselves. Moreover, $(1,0) \leq T(1,0)=(1,0)$ and $T$ has two fixed points in $X$.

In what follows, we give a sufficient condition for the uniqueness of the fixed point in Theorems 2.1 and 2.2. This condition is as follows:

for $x, y \in X$, there exists a lower bound or an upper bound.

In [17], it is proved that the condition (10) is equivalent to

for $x, y \in X$, there exists $z \in X$ which is comparable to $x$ and $y$. 
Theorem 2.3 Adding the condition (11) to the hypotheses of Theorem 2.1 (resp. Theorem 2.2), we obtain the uniqueness of the fixed point of $T$.

Proof Suppose that there exist $z, y \in X$ which are fixed points. We distinguish the following two cases:

Case 1. If $y$ is comparable to $z$, then $T^{n}(y)=y$ is comparable to $T^{n}(z)=z$ for $n=0,1,2, \ldots$ and

$$
\begin{aligned}
\psi(d(z, y)) & =\psi\left(d\left(T^{n}(z), T^{n}(y)\right)\right) \\
& \leq \phi\left(d\left(T^{n-1}(z), T^{n-1}(y)\right)\right) \\
& \leq \phi(d(z, y)) .
\end{aligned}
$$

By the condition $\psi(t)>\phi(t)$ for $t>0$, we obtain $d(z, y)=0$ and this implies $z=y$.

Case 2. If $y$ is not comparable to $z$, then there exists $x \in X$ comparable to $y$ and $z$. Monotonicity of $T$ implies that $T^{n}(x)$ is comparable to $T^{n}(y)$ and to $T^{n}(z)=z$, for $n=0,1,2, \ldots$. Moreover,

$$
\begin{aligned}
\psi\left(d\left(z, T^{n}(x)\right)\right) & =\psi\left(d\left(T^{n}(z), T^{n}(x)\right)\right) \\
& \leq \phi\left(d\left(T^{n-1}(z), T^{n-1}(x)\right)\right) \\
& =\phi\left(d\left(z, T^{n-1}(x)\right)\right) .
\end{aligned}
$$

Hence, $\psi$ is an altering distance function and the condition of $\psi(t)>\phi(t)$ for $t>0$. This gives us that $\left\{d\left(z, f^{n}(x)\right)\right\}$ is a nonnegative decreasing sequence, and consequently, there exists $\gamma$ such that

$$
\lim _{n \rightarrow \infty} d\left(z, T^{n}(x)\right)=\gamma
$$

Letting $n \rightarrow \infty$ in (12) and taking into account that $\psi$ and $\Phi$ are continuous functions, we obtain

$$
\psi(\gamma) \leq \phi(\gamma)
$$

This and the condition of Theorem 2.1 implies $\phi(\gamma)=0$, and consequently, $\gamma=0$.

Analogously, it can be proved that

$$
\lim _{n \rightarrow \infty} d\left(y, T^{n}(x)\right)=0 .
$$

Finally, as

$$
\lim _{n \rightarrow \infty} d\left(z, T^{n}(x)\right)=\lim _{n \rightarrow \infty} d\left(y, T^{n}(x)\right)=0,
$$

the uniqueness of the limit gives us $y=z$. This finishes the proof.

Remark 2.1 Under the assumption of Theorem 2.3, it can be proved that for every $x \in X$, $\lim _{n \rightarrow \infty} T^{n}(x)=z$, where $z$ is the fixed point (i.e., the operator $f$ is Picard). 
Remark 2.2 Theorem 1.1 is a particular case of Theorem 2.1 for $\psi$, the identity function, and $\phi(x)=x-\psi(x)$.

Theorem 1.2 is a particular case of Theorem 2.1 for $\phi(x)=\psi(x)-\phi_{1.2}(x), \phi_{1.2}$ is an altering function in Theorem 1.2. Theorem 1.3 is a particular case of Theorem 2.1 for $\psi$, the identity function, and $\phi(x)=\psi(x) x$.

\section{Application to ordinary differential equations}

In this section, we present two examples where our Theorems 2.2 and 2.3 can be applied. The first example is inspired by [17]. We study the existence of a solution for the following first-order periodic problem:

$$
\left\{\begin{array}{l}
u^{\prime}(t)=f(t, u(t)), \quad t \in[0, T] \\
u(0)=u(T),
\end{array}\right.
$$

where $T>0$ and $f: I \times R \rightarrow R$ is a continuous function. Previously, we considered the space $C(I)(I=[0, T])$ of continuous functions defined on $I$. Obviously, this space with the metric given by

$$
d(x, y)=\sup \{|x(t)-y(t)|: t \in I\}, \quad \text { for } x, y \in C(I)
$$

is a complete metric space. $C(I)$ can also be equipped with a partial order given by

$$
x, y \in C(I), \quad x \leq y \quad \Leftrightarrow \quad x(T) \leq y(t) \quad \text { for } t \in I .
$$

Clearly, $(C(I), \leq)$ satisfies the condition (10) since for $x, y \in C(I)$, the functions $\max \{x, y\}$ and $\min \{x, y\}$ are the least upper and the greatest lower bounds of $x$ and $y$, respectively. Moreover, in [17] it is proved that $(C(I), \leq)$ with the above mentioned metric satisfies the condition (9).

Now, we give the following definition.

Definition 3.1 A lower solution for (13) is a function $\alpha \in C^{(1)}(I)$ such that

$$
\left\{\begin{array}{l}
\alpha^{\prime}(t) \leq f(t, \alpha(t)), \quad \text { for } t \in I, \\
\alpha(0) \leq \alpha(T) .
\end{array}\right.
$$

Theorem 3.1 Consider the problem (13) with $f: I \times R \rightarrow R$ continuous, and suppose that there exist $\lambda, \alpha>0$ with

$$
\alpha \leq\left(\frac{2 \lambda\left(e^{\lambda T}-1\right)}{T\left(e^{\lambda T}+1\right)}\right)^{\frac{1}{2}}
$$

such that for $x, y \in R$ with $x \geq y$,

$$
0 \leq f(t, x)+\lambda x-[f(t, y)+\lambda y] \leq \alpha \sqrt{\ln \left[(x-y)^{2}+1\right]} .
$$

Then the existence of a lower solution for (13) provides the existence of a unique solution of (13). 
Proof The problem (13) can be written as

$$
\left\{\begin{array}{l}
u^{\prime}(t)+\lambda u(t)=f(t, u(t))+\lambda u(t), \quad \text { for } t \in I=[0, T] \\
u(0)=u(T)
\end{array}\right.
$$

This problem is equivalent to the integral equation

$$
u(t)=\int_{0}^{T} G(t, s)[f(s, u(s))+\lambda u(s)] d s
$$

where $G(t, s)$ is the Green function given by

$$
G(t, s)= \begin{cases}\frac{e^{\lambda(T+s-t)}}{e^{\lambda T}-1}, & 0 \leq s<t \leq T, \\ \frac{e^{\lambda(s-t)}}{e^{\lambda T}-1}, & 0 \leq t<s \leq T .\end{cases}
$$

Define $F: C(I) \rightarrow C(I)$ by

$$
(F u)(t)=\int_{0}^{T} G(t, s)[f(s, u(s))+\lambda u(s)] d s .
$$

Note that if $u \in C(I)$ is a fixed point of $F$, then $u \in C^{1}(I)$ is a solution of (13). In what follows, we check that the hypotheses in Theorems 2.2 and 2.3 are satisfied. The mapping $F$ is nondecreasing for $u \geq v$; using our assumption, we can obtain

$$
f(t, u)+\lambda u \geq f(t, v)+\lambda v
$$

which implies, since $G(t, s)>0$, that for $t \in I$,

$$
\begin{aligned}
(F u)(t) & =\int_{0}^{T} G(t, s)[f(s, u(s))+\lambda u(s)] d s \\
& \geq \int_{0}^{T} G(t, s)[f(s, v(s))+\lambda v(s)] d s=(F v)(t) .
\end{aligned}
$$

Besides, for $u \geq v$, we have

$$
\begin{aligned}
d(F u, F v) & =\sup _{t \in I}|(F u)(t)-(F v)(t)| \\
& =\sup _{t \in I}((F u)(t)-(F v)(t)) \\
& =\sup _{t \in I} \int_{0}^{T} G(t, s)[f(s, u(s))+\lambda u(s)-f(s, v(s))-\lambda v(s)] d s \\
& \leq \sup _{t \in I} \int_{0}^{T} G(t, s) \alpha \sqrt{\ln \left[(u(s)-v(s))^{2}+1\right]} d s .
\end{aligned}
$$

Using the Cauchy-Schwarz inequality in the last integral, we get

$$
\begin{aligned}
& \int_{0}^{T} G(t, s) \alpha \sqrt{\ln \left[(u(s)-v(s))^{2}+1\right]} d s \\
& \quad \leq\left(\int_{0}^{T} G(t, s)^{2} d s\right)^{\frac{1}{2}}\left(\int_{0}^{T} \alpha^{2} \ln \left[(u(s)-v(s))^{2}+1\right] d s\right)^{\frac{1}{2}} .
\end{aligned}
$$


The first integral gives us

$$
\begin{aligned}
\int_{0}^{T} G(t, s)^{2} d s & =\int_{0}^{t} G(t, s)^{2} d s+\int_{t}^{T} G(t, s)^{2} d s \\
& =\int_{0}^{t} \frac{e^{2 \lambda(T+s-t)}}{\left(e^{\lambda T}-1\right)^{2}} d s+\int_{t}^{T} \frac{e^{2 \lambda(s-t)}}{\left(e^{\lambda T}-1\right)^{2}} d s \\
& =\frac{1}{2 \lambda\left(e^{\lambda T}-1\right)^{2}}\left(e^{2 \lambda T-1)}\right. \\
& =\frac{e^{\lambda T}+1}{2 \lambda\left(e^{\lambda T}-1\right)} .
\end{aligned}
$$

The second integral in (15) gives us the following estimate:

$$
\begin{aligned}
\int_{0}^{T} \alpha^{2} \ln \left[(u(s)-v(s))^{2}+1\right] d s & \leq \alpha^{2} \ln \left[\|u-v\|^{2}+1\right] \cdot T \\
& =\alpha^{2} \ln \left[d(u, v)^{2}+1\right] \cdot T .
\end{aligned}
$$

Taking into account (14)-(17), we have

$$
\begin{aligned}
d(F u, F v) & \leq \sup _{t \in I}\left(\frac{e^{\lambda T}+1}{2 \lambda\left(e^{\lambda T}-1\right)}\right)^{\frac{1}{2}} \cdot\left(\alpha^{2} \ln \left[d(u, v)^{2}+1\right] \cdot T\right)^{\frac{1}{2}} \\
& =\left(\frac{e^{\lambda T}+1}{2 \lambda\left(e^{\lambda T}-1\right)}\right)^{\frac{1}{2}} \cdot \alpha \cdot \sqrt{T} \cdot\left(\ln \left[d(u, v)^{2}+1\right]\right)^{\frac{1}{2}},
\end{aligned}
$$

and from the last inequality, we obtain

$$
d(F u, F v)^{2} \leq \frac{e^{\lambda T}+1}{2 \lambda\left(e^{\lambda T}-1\right)} \cdot \alpha^{2} \cdot T \cdot \ln \left[d(u, v)^{2}+1\right]
$$

or equivalently,

$$
2 \lambda\left(e^{\lambda T}-1\right) d(F u, F v)^{2} \leq\left(e^{\lambda T}+1\right) \cdot \alpha^{2} \cdot T \cdot \ln \left[d(u, v)^{2}+1\right] .
$$

By our assumption, as

$$
\alpha \leq\left(\frac{2 \lambda\left(e^{\lambda T}-1\right)}{T\left(e^{\lambda T}+1\right)}\right)^{\frac{1}{2}}
$$

the last inequality gives us

$$
2 \lambda\left(e^{\lambda T}-1\right) d(F u, F v)^{2} \leq 2 \lambda\left(e^{\lambda T}-1\right) \cdot \ln \left[d(u, v)^{2}+1\right],
$$

and hence,

$$
d(F u, F v)^{2} \leq \ln \left[d(u, v)^{2}+1\right] .
$$


Put $\psi(x)=x^{2}$ and $\phi=\ln \left(x^{2}+1\right)$. Obviously, $\psi$ is an altering distance function, $\psi(x)$ and $\phi(x)$ satisfy the condition of $\psi(x)>\phi(x)$ for $x>0$. From (18), we obtain for $u \geq v$,

$$
\psi(d(F u, F v)) \leq \phi(d(u, v))
$$

Finally, let $\alpha(t)$ be a lower solution for (13). We claim that $\alpha \leq F(\alpha)$. In fact

$$
\alpha^{\prime}(t)+\lambda \alpha(t) \leq f(t, \alpha(t))+\lambda \alpha(t), \quad \text { for } t \in I \text {. }
$$

Multiplying by $e^{\lambda t}$

$$
\left(\alpha(t) e^{\lambda t}\right)^{\prime} \leq[f(t, \alpha(t))+\lambda \alpha(t)] e^{\lambda t}, \quad \text { for } t \in I,
$$

we get

$$
\alpha(t) e^{\lambda t} \leq \alpha(0)+\int_{0}^{t}[f(s, \alpha(s))+\lambda \alpha(s)] e^{\lambda s} d s, \quad \text { for } t \in I
$$

As $\alpha(0) \leq \alpha(T)$, the last inequality gives us

$$
\alpha(0) e^{\lambda t} \leq \alpha(T) e^{\lambda T} \leq \alpha(0)+\int_{0}^{T}[f(s, \alpha(s))+\lambda \alpha(s)] e^{\lambda s} d s
$$

and so

$$
\alpha(0) \leq \int_{0}^{T} \frac{e^{\lambda s}}{e^{\lambda T}-1}[f(s, \alpha(s))+\lambda \alpha(s)] d s
$$

This and (19) give us

$$
\alpha(t) e^{\lambda t} \leq \int_{0}^{t} \frac{e^{\lambda(T+s)}}{e^{\lambda T}-1}[f(s, \alpha(s))+\lambda \alpha(s)] d s+\int_{t}^{T} \frac{e^{\lambda s}}{e^{\lambda T}-1}[f(s, \alpha(s))+\lambda \alpha(s)] d s
$$

and consequently,

$$
\begin{aligned}
\alpha(t) & \leq \int_{0}^{t} \frac{e^{\lambda(T+s-t)}}{e^{\lambda T}-1} d s+\int_{0}^{t} \frac{e^{\lambda(s-t)}}{e^{\lambda T}-1}[f(s, \alpha(s))+\lambda \alpha(s)] d s \\
& =\int_{0}^{T} G(t, s)[f(s, \alpha(s))+\lambda \alpha(s)] d s \\
& =(F \alpha)(t), \quad \text { for } t \in I .
\end{aligned}
$$

Finally, Theorems 2.2 and 2.3 give that $F$ has an unique fixed point.

The second example where our results can be applied is the following two-point boundary value problem of the second-order differential equation

$$
\left\{\begin{array}{l}
-\frac{d^{2} x}{d t^{2}}=f(t, x), \quad x \in[0, \infty], t \in[0,1] \\
x(0)=x(1)=0
\end{array}\right.
$$


It is well known that $x \in C^{2}[0,1]$, a solution of $(20)$, is equivalent to $x \in C[0,1]$, a solution of the integral equation

$$
x(t)=\int_{0}^{1} G(t, s) f(s, x(s)) d s, \quad \text { for } t \in[0,1]
$$

where $G(t, s)$ is the Green function given by

$$
G(t, s)= \begin{cases}t(1-s), & 0 \leq t \leq s \leq 1 \\ s(1-t), & 0 \leq s \leq t \leq 1\end{cases}
$$

Theorem 3.2 Consider the problem (20) with $f: I \times R \rightarrow[0, \infty)$ continuous and nondecreasing with respect to the second variable, and suppose that there exists $0 \leq \alpha \leq 8$ such that for $x, y \in R$ with $y \geq x$,

$$
f(t, y)-f(t, x) \leq \alpha \sqrt{\ln \left[(y-x)^{2}+1\right]} .
$$

Then our problem (20) has a unique nonnegative solution.

Proof Consider the cone

$$
P=\{x \in C[0,1]: x(t) \geq 0\} .
$$

Obviously, $(P, d)$ with $d(x, y)=\sup \{|x(t)-y(t)|: t \in[0,1]\}$ is a complete metric space. Consider the operator given by

$$
(T x)(t)=\int_{0}^{1} G(t, s) f(s, x(s)) d s, \quad \text { for } x \in P
$$

where $G(t, s)$ is the Green function appearing in (21).

As $f$ is nondecreasing with respect to the second variable, then for $x, y \in P$ with $y \geq x$ and $t \in[0,1]$, we have

$$
(T y)(t)=\int_{0}^{1} G(t, s) f(s, y(s)) d s \geq \int_{0}^{1} G(t, s) f(s, x(s)) d s \geq(T x)(t),
$$

and this proves that $T$ is a nondecreasing operator.

Besides, for $y \geq x$ and taking into account (22), we can obtain

$$
\begin{aligned}
d(T y, T x) & =\sup _{t \in[0,1]}|(T y)(t)-(T x)(t)| \\
& =\sup _{t \in[0,1]}((T y)(t)-(T x)(t)) \\
& =\sup _{t \in[0,1]} \int_{0}^{1} G(t, s)(f(s, y(s))-f(s, x(s))) d s \\
& \leq \sup _{t \in[0,1]} \int_{0}^{1} G(t, s) \alpha \sqrt{\ln \left[(y-x)^{2}+1\right]}
\end{aligned}
$$




$$
\begin{aligned}
& \leq \sup _{t \in[0,1]} \int_{0}^{1} G(t, s) \alpha \sqrt{\ln \left[\|y-x\|^{2}+1\right]} d s \\
& =\alpha \sqrt{\ln \left[\|y-x\|^{2}+1\right]} \sup _{t \in[0,1]} \int_{0}^{1} G(t, s) d s .
\end{aligned}
$$

It is easy to verify that

$$
\int_{0}^{1} G(t, s) d s=\frac{-t^{2}}{2}+\frac{t}{2}
$$

and that

$$
\sup _{t \in[0,1]} \int_{0}^{1} G(t, s) d s=\frac{1}{8} .
$$

These facts, the inequality (23), and the hypothesis $0<\alpha \leq 8$ give us

$$
\begin{aligned}
d(T y, T x) & \leq \frac{\alpha}{8} \sqrt{\ln \left[\|y-x\|^{2}+1\right]} \\
& \leq \sqrt{\ln \left[\|y-x\|^{2}+1\right]}=\sqrt{\ln \left[d(x, y)^{2}+1\right]}
\end{aligned}
$$

Hence

$$
d(T y, T x)^{2} \leq \ln \left[d(x, y)^{2}+1\right]
$$

Put $\psi(x)=x^{2}, \phi(x)=\ln \left(x^{2}+1\right)$; obviously, $\psi$ is an altering distance function, $\psi$ and $\phi$ satisfy the condition $\psi(x)>\phi(x)$, for $x>0$. From the last inequality, we have

$$
\psi(d(T x, T y)) \leq \phi(d(x, y))
$$

Finally, as $f$ and $G$ are nonnegative functions,

$$
(T 0)(t)=\int_{0}^{1} G(t, s) f(s, 0) d s \geq 0 .
$$

Theorems 2.2 and 2.3 tell us that $F$ has a unique nonnegative solution.

\section{Competing interests}

The authors declare that they have no competing interests.

\section{Authors' contributions}

All the authors contributed equally to the writing of the present article. All authors read and approved the final manuscript.

\section{Acknowledgements}

This project is supported by the National Natural Science Foundation of China under the grant (11071279).

Received: 28 April 2012 Accepted: 30 August 2012 Published: 18 September 2012 


\section{References}

1. Khan, MS, Swaleh, M, Sessa, S: Fixed point theorems by altering distances between the points. Bull. Aust. Math. Soc 30(1), 1-9 (1984)

2. Dhutta, P, Choudhury, B: A generalization of contractions in partially ordered metric spaces. Appl. Anal. 87, 109-116 (2008)

3. Rhoades, BE: Some theorems on weakly contractive maps. Nonlinear Anal. 47, 2683-2693 (2001)

4. Aydi, H, Karapnar, E, Bessem, S: Fixed point theorems in ordered abstract spaces. Fixed Point Theory Appl. 2012,76 (2012)

5. Nieto, JJ, Pouso, RL, Rodríguez-López, R: A fixed point theorem for contraction type maps in partially ordered metric spaces and application to ordinary differential equations. Proc. Am. Math. Soc. 135, 2505-2517 (2007)

6. Gordji, ME, Baghani, H, Kim, GH: Coupled fixed point theorems for contraction mapping induced by cone ball-metric in partially ordered spaces. Discrete Dyn. Nat. Soc. 2012, art. no. 981517 (2012)

7. Sintunavarat, W, Cho, YJ, Kumam, P: Existence, regularity and stability properties of periodic solutions of a capillarity equation in the presence of lower and upper solutions. Fixed Point Theory Appl. 2012, 128 (2012)

8. Obersnel, F, Omari, P, Rivetti, S: A generalization of Geraghty's theorem in partially ordered metric spaces and applications to ordinary differential equations. Nonlinear Anal., Real World Appl. 13, 2830-2852 (2012)

9. Sastry, K, Babu, G: Some fixed point theorems by altering distance between the points. Indian J. Pure Appl. Math. 30 641-647 (1999)

10. Amini-Harandi, A, Emami, H: A fixed point theorem for contraction type maps in partially ordered metric spaces and application to ordinary differential equations. Nonlinear Anal. 72, 2238-2242 (2010)

11. Harjani, J, Sadarangni, K: Fixed point theorems for weakly contraction mappings in partially ordered sets. Nonlinear Anal. 71, 3403-3410 (2009)

12. Burgic, D, Kalabusic, S, Kulenovic, M: Global attractivity results for mixed monotone mappings in partially ordered complete metric spaces. Fixed Point Theory Appl. 2009, Article ID 762478 (2009)

13. Ciric, L, Cakid, N, Rajovic, M, Uma, J: Monotone generalized nonlinear contractions in partially ordered metric spaces. Fixed Point Theory Appl. 2008, Article ID 131294 (2008)

14. Gnana Bhaskar, T, Lakshmikantham, V: Fixed point theorems in partially ordered metric spaces and applications. Nonlinear Anal. 65, 1379-1393 (2006)

15. Lakshmikantham, V, Ciric, L: Coupled fixed point theorems for nonlinear contractions in partially ordered metric spaces. Nonlinear Anal. 70, 4341-4349 (2009)

16. Nieto, JJ, Rodriquez-López, R: Contractive mapping theorems in partially ordered sets and applications to ordinary differential equations. Order 22, 223-239 (2005)

17. Nieto, JJ, Rodriquez-López, R: Existence and uniqueness of fixed point in partially ordered sets and applications to ordinary differential equations. Acta Math. Sin. 23, 2205-2212 (2007)

18. O'Regan, D, Petrusel, A: Fixed point theorems for generalized contractions in ordered metric spaces. J. Math. Anal. Appl. 341, 1241-1252 (2008)

19. Harjani, J, Sadarangni, K: Generalized contractions in partially ordered metric spaces and applications to ordinary differential equations. Nonlinear Anal. 72, 1188-1197 (2010)

\section{Submit your manuscript to a SpringerOpen ${ }^{\circledR}$ journal and benefit from:}

- Convenient online submission

- Rigorous peer review

Immediate publication on acceptance

Open access: articles freely available online

- High visibility within the field

- Retaining the copyright to your article 